\title{
Auricular Acupuncture Therapy for Stage I of Shoulder Hand Syndrome (SHS) After Ischemic Stroke: Study Protocol for a Randomized, Sham- controlled, Patient-blinded, Clinical Trial
}

Jie Sun

Beijing Xiaotangshan Hospital

Xiaonan Meng ( $\nabla$ mengxiaonan.cn@gmai.com )

Beijing Huguosi TCM Hospital, affiliated to Beijing University of Chinese Medcine https://orcid.org/00000002-3505-1532

\section{Terry Oleson}

Emperor's College of Traditional Oriental Medicine

\section{Liping Wang}

Beijing Huguosi TCM Hospital, affiliated to BUCM

\section{Chunying Li}

Beijing Huguosi TCM Hospital, affiliated to BUCM

\section{Lufen Zhang}

Beijing University of Chinese Medicine

\section{Haikuo Yu}

Xuanwu Hospital

\section{Zhen Zhao}

Beijing Huguosi TCM Hospital, affiliated to BUCM

\section{Sen Gao}

Beijing Huguosi TCM Hospital, affiliated to BUCM

\section{Study protocol}

Keywords: Auricular acupuncture, Shoulder hand syndrome, Randomized controlled trial, Study protocol

Posted Date: May 10th, 2021

DOI: https://doi.org/10.21203/rs.3.rs-44269/v1

License: (1) (1) This work is licensed under a Creative Commons Attribution 4.0 International License. Read Full License 


\section{Abstract}

\section{Background:}

Shoulder hand syndrome (SHS) is one of the common complications after a stroke, with the incidence rate up to $75 \%$ in China. The stage $\mathbb{Q}$ level is manifested as chronic pain and limitation of movement, which could cause irreversible muscle atrophy, joint deformation and even loss of motor function if progress to

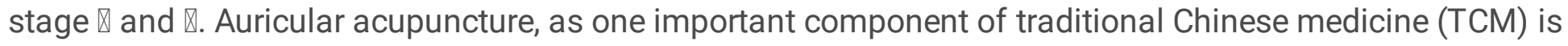
a micro acupuncture therapy which combines acupuncture and holographic theory. However, the clinical efficacy of auricular acupuncture is still controversial and the length of improvement by auricular acupuncture has not been validated by clinical studies. Therefore, this study aims to evaluate the efficacy and duration of auricular acupuncture for pain relief and for motor function improvement in stage $₫$ of SHS after ischemic stroke.

\section{Methods:}

This is a randomized, sham-controlled, patient-blinded trial. A total of 108 eligible patients with stage $\nabla$ of SHS after ischemic stroke will be recruited from September 2018 to December 2020 and will be randomly allocated to three groups, A, B, or C, with a 1:1:1 ratio. Only body acupuncture will be applied in group $A$ as a control group. Non-needle-insertion for sham auricular acupuncture will be combined with body acupuncture and applied to group B, whereas auricular acupuncture combined with body acupuncture will be applied in group C. Patients will be treated for 30 min per session, for 5 sessions per week for 3 weeks, and followed up for 6 weeks. The primary outcome will be the visual analog scale (VAS) for the shoulder and hand pain. The secondary outcomes will include the Fugl-Meyer Assessment of the Upper Extremity (FMA-UE) and Modified Brathel Index (MBI). Eligible patients would be evaluated before treatment, immediately after treatment, and followed up after 1 week, 2 weeks and 6 weeks to verify the duration of auricular acupuncture effect.

\section{Discussion:}

This trial will help to identify the effectiveness and duration of auricular acupuncture effect for stage $\nabla$ of SHS after ischemic stroke.

\section{Trial registration:}

www.chictr.org.cn, Chinese Clinical Trial Registry: ChiCTR2000037946. Retrospectively registered on 4 March 2020.

\section{Background}

Stroke is the second leading cause of death and one of the main causes of disability worldwide [1], with a mortality rate that is higher in low-and medium-income countries [2]. Globally, neurological disorders were the leading cause of disability-adjusted life-years (DALYs) in 2016, and stroke was the largest contributors of neurological DALYs [2-3]. In 2005 there were more than 10 million stroke patients in China, with a rapid 
annual growth rate of $8.7 \%$ [4]. The incidence rate of stroke has been rising significantly, due to the aging population of China since 2010 and is expected to increase by about $50 \%$ in 2030 [5]. In 2016, the number of stroke deaths is nearly 2 million, and the mortality rate is $140.3 / 100000$, accounting for $22.8 \%$ of the total deaths in China [6]. Shoulder hand syndrome (SHS), as one of the most common complications after stroke, was firstly reported by Oscar Stejnbroker in 1947. It is also called reflex sympathetic dystrophy (RSD) because of the relation with the sympathetic nervous system dysfunction affecting the upper limbs [7]. Its incidence rate varies from different regions in China, from $10-75 \%$, and the age of its incidence is between $45-78$ years old [8]. The stage $\nabla$ of SHS is mainly manifested as the regional joint pain with limited range of activity, which can progress to stage $\otimes$ and $\otimes$, leading to the irreversible muscle atrophy, joint deformation or even complete loss of motor function [9].

There is no consensus on the clinical treatment guidelines for SHS. The treatment can be pharmacological, non-pharmacological, such as physiotherapy and psychotherapy, or surgical, such as regional anesthesia and sympathectomy [10], but the clinical effect is far from satisfaction. Acupuncture, as an important part of traditional Chinese medicine (TCM), has been effectively applied to improve pain disorders and motor dysfunction [11]. As one component of acupuncture, auricular acupuncture (AA) is a micro acupuncture therapy which combines meridian acupuncture with holographic theory, wherein one part of the body represents the whole of the body. However, there are neither unified acupuncture treatment principles nor standardized treatment protocols for SHS, nor are there solid clinical trial results to testify the clinical efficacy of AA on SHS, making its effect controversial [12-13]. Hence, there is a need for rigorous clinical randomized controlled trials (RCTs) in order to validate the AA effect for SHS. In this study, the shamcontrolled, patient-blinded RCT will be applied with the purpose of evaluating the clinical efficacy and duration of AA in the treatment of SHS after ischemic stroke.

\section{Methods}

\section{Trial design}

In this study, the randomized sham-controlled, patient-blinded trial is designed with in a single clinical center. The trial is being conducted in Beijing Huguosi TCM Hospital, affiliated to Beijing University of Chinese Medicine (BUCM), with the assistance of Beijing Xiaotangshan Hospital and Xuanwu Hospital Capital Medical University. All three hospitals are located in Beijing, China. The trial has been started on 1 September 2018 and will be closed on 31 December 2020. This protocol has been approved by the Medical Ethics Committee of Beijing Huguosi TCM Hospital, affiliated to BUCM (No. HZ-20180518), and will follow the principles of Declaration of Helsinki. This trial was registered on 4 March 2020 in Chinese Clinical Trial Registry with the number of ChiCTR2000037946.

Prior to their participation, all patients will be informed about the purpose of the study, the procedure and the related risks in detail. The eligible patients are required to sign a written informed consent, and all participants have the right to withdraw unconditionally from the study at any time. 108 eligible patients with SHS after ischemic stroke will be recruited and randomly allocated into the groups A, B, or C, with a 
1:1:1 ratio (Fig. 1): body acupuncture (BA) applied (group A), sham AA combined with BA (group B), or AA combined with BA (group C).

\section{Inclusion criteria}

Participants will be included in accordance with following criteria: (1) conformed to the diagnostic standards of cerebral infarction [14] by cerebral imaging (including but not limited to CT or MRI); (2) diagnosed with SHS stage I [15]; (3) the first or second time of ischemic stroke onset; (4) treated after the onset between two weeks and three months; (5) gender unlimited; (5) the age between 50-76 years old; (6) stable vital signs; (7) full awareness of this study and agreement on signing the informed consent form.

\section{Exclusion criteria}

Participants will be excluded if they conformed to any of the following items: (1) any cerebrovascular diseases other than ischemic stroke; (2) patients with abnormal pain threshold caused by thalamus infarction; (3) unstable vital signs; (4) patients with serious mental disorders after ischemic stroke; (5) more than three times of stroke onset; (6) patients with dislocation and subluxation of shoulder joint; (7) patients with peri-arthritis of shoulder and local soft tissue injury before stroke; (8) intolerance of treatment or refusal to sign the informed consent form.

\section{Participant recruitment}

Participants in this study will be recruited through the way as follows: (1) on-line media, such as the official website or WeChat platform of Beijing Huguosi TCM Hospital, affiliated to BUCM, (2) off-line advertising, such as flyers or bulletin boards, placing at Acupuncture Department and Rehabilitation Department of Beijing Huguosi TCM Hospital, affiliated to BUCM, Beijing Xiaotangshan Hospital and Xuanwu Hospital Capital Medical University. Any potential participants can contact recruitment staffs through provided phone or WeChat number and only those who meet the inclusion criteria and are willing to sign the informed consent form can be participated into this study. The trial schedule is shown in Fig. 1.

\section{Randomization and allocation}

An independent researcher will create a randomization sequence according to the method of random number table, which will be hidden in an opaque-and-sealed envelope. The 108 eligible participants will be allocated into groups $A, B$, or $C$, with a 1:1:1 ratio. After baseline evaluation and following signature of informed consent form, the research assistants will open an envelope with the randomization number for the patient, and the patients will only be informed of their randomization number. At the same time, the researchers who will provide treatment will be informed of the randomization number and allocation through email or WeChat platform. There will be a backup of the randomization sequence kept by the Clinical Research Monitoring Department (CRMD) of Beijing Huguosi TCM Hospital, affiliated to BUCM to avoid the intentional group switch (Fig. 2).

\section{Blinding}


It is unavoidable for the clinical researchers conducting the acupuncture interventions to not be informed of the treatment procedure because this research will be conducted as a procedure-related interventional RCT. However, all participants will remain blinded to the allocation of their treatment throughout the study. Seirin Pyonex Needles (made by Seirin Corporation in Japan), with the size of $0.20 \mathrm{~mm}$ (diameter) $\times 1.2 \mathrm{~mm}$ (length), will be applied as auricular needles in this trial. The auricular needle with the adhesive plaster as the foundation, will be embedded into the auricular point without any sensation of insertion due to the small needle size applied in group $\mathrm{C}$. The sham auricular acupuncture will be prepared by removal of the auricular needles prior to the intervention, without any notice of participants in group B since there will not be sensation either and they will be totally identical with group C from the exterior (Fig. $\underline{\mathbf{3}}$ ). The rehabilitation physicians who will evaluate the outcomes and statistician who will analyze the outcomes, will also be blinded to the allocation.

\section{Interventions}

All participants will be closely monitored for the risk factors related to stroke, such as blood pressure, blood glucose, blood lipid, homocysteine, et al, and be maintained by proper medications. For the BA treatment, acupoints will be selected as follows: LI 15 (Jianyu), SJ 14 (Jianliao), SI 9 (Jianzhen), SJ 5 (Waiguan), LI 4 (Hegu) and SI 3 (Houxi), all of which are located on the affected side of the body (Table 1). The disposable sterile stainless-steel filiform needles (made by Beijing Keyuanda Medical Device Manufactory) will be applied with the size of $0.25 \mathrm{~mm}$ (diameter) $\times 40 \mathrm{~mm}$ (length). With regional skin fully exposed and disinfected, the filiform needles will be inserted vertically into the acupoints for $25 \mathrm{~mm}$, with the evenmethod hand manipulation.

For participants in group $\mathrm{B}$ and $\mathrm{C}$, auricular acupoints will be selected according to the auricular standardization [16] issued by the World Federation of Acupuncture- Moxibustion Societies (WFAS) in May 2013. The acupoints are as follows (Table 2): $\mathrm{SF}_{1}$ (finger), $\mathrm{SF}_{2}$ (wrist), $\mathrm{SF}_{3}$ (elbow), $\mathrm{SF}_{4,5}$ (shoulder), $\mathrm{AH}_{6}$ a (sympathetic nerve) and $\mathrm{AT}_{4}$ (subcortex). The Seirin Pyonex Needles, the disposable sterile auricular needles, with the size of $0.2 \mathrm{~mm}$ (diameter) $\times 1.2 \mathrm{~mm}$ (length), will be inserted into the designated auricle acupoints, and then fixed by the adhesive plaster on the foundation in group $\mathrm{C}$. The intervention in group $\mathrm{B}$ will be exactly the same as that of in group $C$, but with the sham auricular needles, which will be removed in advance without any notice or sense of participants. The appearances of the auricular needles in two groups will be identical. All these interventions will be performed for $30 \mathrm{~min}$ per session, for 5 sessions per week for 3 weeks. 
Table 1

Protocol for acupoints

\begin{tabular}{|c|c|}
\hline Acupoints & Location \\
\hline $\begin{array}{l}\text { LI } 15 \\
\text { (Jianyu) }\end{array}$ & $\begin{array}{l}\text { In the shoulder region, in the deltoid depression when the arm is abducted or extended } \\
\text { forward. }\end{array}$ \\
\hline $\begin{array}{l}\text { SJ } 14 \\
\text { (Jianliao) }\end{array}$ & $\begin{array}{l}\text { In the shoulder region, in the posterior depression of shoulder peak when the arm is } \\
\text { abducted. }\end{array}$ \\
\hline $\begin{array}{l}\text { SI } 9 \\
\text { (Jianzhen) }\end{array}$ & $\begin{array}{l}\text { In the shoulder region, } 1 \text { cun above the posterior crease of armpit when the arm is } \\
\text { adducted. }\end{array}$ \\
\hline $\begin{array}{l}\text { SJ } 5 \\
\text { (Waiguan) }\end{array}$ & $\begin{array}{l}\text { Right in the middle of the crevice between the ulna and radius, and } 2 \text { cun above the } \\
\text { dorsal carpal striation. }\end{array}$ \\
\hline $\begin{array}{l}\text { LI } 4 \\
\text { (Hegu) }\end{array}$ & $\begin{array}{l}\text { In the region of dorsum of hand, on the middle point of the radial side of the second } \\
\text { metacarpal bone. }\end{array}$ \\
\hline $\begin{array}{l}\text { SI } 3 \\
\text { (Houxi) }\end{array}$ & $\begin{array}{l}\text { In the red-and-white fleshy border of ulnar side, on the proximal metacarpal transverse } \\
\text { crease of the fifth metacarpal joint. }\end{array}$ \\
\hline
\end{tabular}

LI large intestine, SJ Sanjiao, SI small intestine

Table 2

Protocol for auricular acupoints

\begin{tabular}{|c|c|}
\hline $\begin{array}{l}\text { Auricular } \\
\text { Acupoints }\end{array}$ & Location \\
\hline $\mathrm{SF}_{1}$ (finger) & In the superior scapha. \\
\hline $\mathrm{SF}_{2}$ (wrist) & In the area inferior to $\mathrm{SF}_{1}$. \\
\hline $\mathrm{SF}_{3}$ (elbow) & In the area inferior to SF2 \\
\hline $\mathrm{SF}_{4,5}$ (shoulder) & In the area inferior to SF3. \\
\hline $\begin{array}{l}\mathrm{AH}_{6} \text { a } \\
\text { (sympathetic } \\
\text { nerve) }\end{array}$ & $\begin{array}{l}\text { At the juncture of the end of the inferior antihelix crus and the medial edge of the } \\
\text { helix. }\end{array}$ \\
\hline $\mathrm{AT}_{4}$ (subcortex) & On the medial side of the antitragus. \\
\hline
\end{tabular}

SF scapha, AH antihelix, AT antitragus

\section{Adverse events}

All participants will be inquired about any discomfort or unexpected signs or symptoms which could be related to the AA application. All the possible adverse events (AEs), which will be closely monitored and documented during the whole course of the trial, are categorized into 3 levels as mild, moderate and severe. 
The mild level means AEs will be temporary and tolerable, the moderate level means that unpleasant symptoms will occur and participant's daily life will be affected and the severe level means that participant's vital signs will be jeopardized or even lead to the risk of life. Any participant with severe AEs will be excluded and provided with immediate medical aid.

\section{Quality control}

This study is designed and revised after consulting the experts of acupuncture, rehabilitation, neurology, methodology and statistics. All researchers participated into this trial will receive trainings on protocol, participant recruitment, intervention, efficacy assessment and data recording. During the whole progress, all data collected will be monitored by the CRMD of Beijing Huguosi TCM Hospital, affiliated to BUCM, which is totally independent and has no conflict of interests. In this study, the case report forms (CRFs) will be applied to record the clinical documents, including the medical history, baseline data, intervention, outcome assessments, follow-up assessments, adverse events and dropouts. Any original data change in paper CRFs should be approved and authorized by CRMD with the justified reason. All the interventions will be performed by attending physicians who have been practicing acupuncture for more than 6 years, and all the outcome measurements will be assessed by rehabilitation physicians with more than 5 years' working experience.

\section{Outcome measurements}

All the outcomes will be assessed before treatment (baseline), immediately after treatment, and follow-ups of 1 week, 2 weeks and 6 weeks.

\section{Primary outcome measurement}

The pain is the most common symptom of patients with SHS [10]. The VAS scores will be adopted as the primary outcome measurement due to its wide application for pain evaluation [17]. The VAS score system consists of a straight line with $100 \mathrm{~mm}$, with two distinct end points, representing the two extremities which stand for " 0 " and " 10 " respectively. The " 0 " indicates "completely no pain" and the " 10 " indicates "the most severe pain that is unbearable". Therefore, participants will mark a point on the straight line that would represent their own pain level.

\section{Secondary outcome measurements}

The Fugl-Meyer Assessment of the Upper Extremity (FMA-UE) will be used to evaluate the motor functions of the upper extremity on the affected side [18]. The FMA-UE is divided into motor assessment and nonmotor assessment. The motor assessment includes function of the hand, forearm and upper arm, evaluated from the fingers to the shoulder. The non-motor assessment includes dermal sensation, pain assessment of passive movement and the range of motion of joints on upper limb. There are 33 items for FMA-UE in total, with a full score of 66 points and each item scored 0-2 points. There are 36 points as full score for forearm and upper arm, and 30 points as full score for the wrist and hand. A higher score represents a better upper extremity motor function. 
The Modified Brathel Index (MBI), based on the Brathel Index (BI) [19], is the measurement of independence on activities of daily living (ADL) and usually applied for stroke patients [20-21]. In MBI, the original rating system has been replaced by a new scale of 5-point system, which is set 100 points as the full score. Based on modified measurement, the higher score indicates the better daily living capability.

\section{Dropouts}

During the course of this trial, any dropout will be monitored and recorded on the CRFs, including the specific reasons and date.

\section{Sample size}

In this study, the VAS is selected as the primary outcome measurement for SHS treatment [22-25]. Based on a previous study [25], it showed that after treatment the VAS ( $\overline{\mathrm{x}} \pm \mathrm{s}$ ) in the body acupuncture group decreased to $3.77 \pm 1.58$. Hence, the calculation for sample size is based on the method of a conventional RCT research [26-27]. Set the significance levl $\alpha=0.05, \beta=0.20$ with the power of $80 \%(1-\beta)$, equal proportion among all groups and the minimum sample size should be 69 (23 for each group), assuming the sample drop rate of $10 \%$. The final sample size is expanded to 108 in total (36 for each group) with a 1:1:1 ratio.

\section{Statistical analysis}

The intention-to-treat principle will be applied for the data analysis, and any missing data will be informed to an independent statistician who will be responsible for statistical calculations and find the appropriate solution. All the original data will be analyzed by the SPSS software (Version 21.0). The categorical data, such as genders in each group will be analyzed as proportion by chi-squared or Fisher's exact test. Continuous variables will be presented by the form of mean \pm standard deviation (SD) in each group. If the continuous variables are with a normal distribution, the analysis of variance (ANOVA) will be adopted. While Kruskal-Wallis $\mathrm{H}$ test will be applied if continuous variables are with a non-normal distribution. The confidence interval will be set at $95 \%$, with the significance level of $5 \%(P<0.05)$.

\section{Discussion}

At present time, there is no consensus on the pathogenesis of SHS after stroke, but its high incidence rate has caused enormous burden to patients and society [2-3]. It is a great challenge for clinicians to avoid the deterioration of SHS from stage $\otimes$ to stage $\otimes[28]$. As an important part of acupuncture, the AA plays an irreplaceable role in pain relief. According to the previous two studies, the AA could even greatly reduce the cancer pain [29-30].

Due to the insufficient numbers of clinical studies on AA and few reports on AA research for SHS, its clinical efficacy is still controversial or underestimated. Therefore, more solid clinical evidences are needed. The non-auricular points were often applied as the sham-controlled group in the previous studies. However, there is still a great controversy about the location of some auricular points worldwide. Therefore, the non- 
auricular points without the global recognition would be easily questioned in clinical practice. In this study, we established and developed the matured clinical sham auricular acupuncture needles as the shamcontrolled group, will select the universal recognized auricular acupoints from another completely different new angle, in order to provide objective clinical evidence. At the same time, different follow-up timepoints will be set to evaluate the clinical efficacy and duration of AA. This trial has been being carried out in Beijing, where receiving the BA treatment after stroke is becoming a common practice in China. Hence the compliance of patients will be greatly reduced, and the ethics committee will not be approval for the study protocol if only AA treatment is provided for SHS after stroke. Therefore, all participants recruited will receive the same BA treatment and the clinical effect of $A A$ will be further evaluated on the basis of $B A$. At the same time, BA group as the control will provide solid clinical evidence for the true efficacy on SHS. This study is a patient-blinded trial, and in order to avoid bias in the treatment process, the rehabilitation physicians who are responsible for the outcome evaluation and statistician who will be charge in data analysis, will be completely blinded to the allocation.

There are limitations in this trial. The first one is physicians who provide interventions will not be blind because the sham auricular needles are easily to be recognized during the insertion into the auricular points. The second limitation is that the patient recruitment is single-centered, and all participants in the trial are Chinese. Therefore, the clinical results may not be universal for other races or countries. The last one is that follow-ups are set at 6 weeks at most, and only the short-term effect of AA can be observed. The medium and long-term effect will not be available from the data of this study.

\section{Trial Status}

This trial has started the participant recruitment from 1 September 2018 and expected to be closed on 31 December 2020. This protocol is version 2.0. 2020-02-17.

\section{Abbreviations}

SHS: shoulder hand syndrome; TCM: traditional Chinese Medicine; VAS: visual analog scale; FMA-UE: FuglMeyer Assessment of the Upper Extremity; MBI: Modified Brathel Index; DALYs: disability-adjusted life-years; RSD: reflex sympathetic dystrophy; AA: auricular acupuncture; BA: body acupuncture; RCTs: randomized controlled trials; BUCM: Beijing University of Chinese Medicine; CRMD: Clinical Research Monitoring Department; CRFs: case report forms; BI: Brathel Index; ADL: activities of daily living.

\section{Declarations}

\section{Acknowledgements}

All authors would like to thank the Nurse Jing Wang and Nurse Xing Tian for their dedications to this study and all the participants enrolled in this trial.

\section{Authors' contributions}


SJ designed the trial, drafted the manuscript and will carry out this trial as one of program coordinators. MXN participated into this trial design, helped with the manuscript and will be the major assistance to conduct the trial as the other program coordinator. And the first two authors contributed equally to this work. TO, as the auricular acupuncture expert, provided professional advice for trial design and revised the manuscript. WLP helped with the manuscript and will be in charge of participant enrollment and all interventions. LCY contributed to the design of trial and will provide interventions for participants. ZLF was consulted as a methodology expert and provided valuable advice and feedback on this trial. YHK will be responsible for data analysis. ZZ helped with interventions on participants and data collection. GS provided rehabilitation therapy and will assess the final outcomes. All authors read and approved this final manuscript.

\section{Funding}

This study is funded by Xicheng District Health Commission of Beijing (No. XWKX2018-11) and Organization Department of Beijing Municipal Committee (No. 2018-81). The funders mentioned above had no role in the study design, data collection, analysis, interpretation or manuscript writing.

\section{Availability of data and materials}

All the related data about this study is available from the corresponding author with the proper request.

\section{Ethics approval and consent to participate}

This protocol has been approved by Medical Ethics Committee of Beijing Huguosi TCM Hospital, affiliated to BUCM (No. HZ-20180518). Prior to the enrollment, participants will be informed about the study, and eligible participants will be required to sign a written informed consent.

\section{Consent for publication}

All participants who signed the informed consent form indicate that they agree with the data publication. The final manuscript has been reviewed by all authors and achieved the consent for publication.

\section{Competing interests}

There are no competing interests declared by all authors.

\section{References}

1. Katan M, Luft A. Global Burden of Stroke. Semin Neurol. 2018;38(2):208-211. doi:10.1055/s-00381649503

2. GBD 2015 Neurological Disorders Collaborator Group. Global, regional, and national burden of neurological disorders during 1990-2015: a systematic analysis for the Global Burden of Disease Study 2015. Lancet Neurol. 2017;16(11):877-897. doi:10.1016/S1474-4422(17)30299-5 
3. GBD 2016 Neurology Collaborators. Global, regional, and national burden of neurological disorders, 1990-2016: a systematic analysis for the Global Burden of Disease Study 2016. Lancet Neurol. 2019;18(5):459-480. doi:10.1016/S1474-4422(18)30499-X

4. World Health Organization. Preventing chronic diseases: a vital investment. WHO Global Report. Geneva. WHO. https://www.who.int/chp/chronic_disease_report/en/. Accessed 2005.

5. Zhang T. Chinese rehabilitation guidelines for stroke, 2011 full version (article in Chinese). Chin J Rehabil Theory Pract. 2012,18(04):301-318.

6. National Health Commission of the People's Republic of China. Interpretation of Work Plan for Comprehensive Prevention and Treatment of Stroke (report in Chinese).

Beijing.NHC.http://www.nhc.gov.cn/jkj/s5879/201612/620d7b78a73b40d098055742336365dc.shtml. Accessed 9 December 2016.

7. Hansson GK. Inflammation, atherosclerosis, and coronary artery disease. N Engl J Med. 2005;352(16):1685-1695. doi:10.1056/NEJMra043430

8. Wang LD, Liu JM, Yang Y, Peng B, Wang YL, et al. Summary of China Stroke Prevention report 2017 (article in Chinese). Chin J Cerebrovasc Dis. 2018,15(11):611-617.

9. Yamanaka $\mathrm{H}$, Yamanaka $\mathrm{H}$. Relationship between cutaneous temperature and hand edema and allodynia after stroke-the etiology of shoulder-hand syndrome (article in Japanese). Rinsho Shinkeigaku. 2015;55(1):1-7. doi:10.5692/clinicalneurol.55.1

10. Pertoldi S, Di Benedetto P. Shoulder-hand syndrome after stroke. A complex regional pain syndrome. Eura Medicophys. 2005;41(4):283-292.

11. Peng L, Zhang C, Zhou L, Zuo HX, He XK, Niu YM. Traditional manual acupuncture combined with rehabilitation therapy for shoulder hand syndrome after stroke within the Chinese healthcare system: a systematic review and meta-analysis. Clin Rehabil. 2018;32(4):429-439. doi:10.1177/0269215517729528

12. Asher GN, Jonas DE, Coeytaux RR, et al. Auriculotherapy for pain management: a systematic review and meta-analysis of randomized controlled trials. J Altern Complement Med. 2010;16(10):10971108. doi:10.1089/acm.2009.0451

13. McGeeney BE. Acupuncture is all placebo and here is why. Headache. 2015;55(3):465-469. doi:10.1111/head.12524

14. Guidelines writing group of diagnosis and treatment for acute ischemic stroke of cerebrovascular disease group of Chinese Society of Neurology of Chinese Medical Association. Chinese guidelines for diagnosis and management of acute ischemic stroke 2010 (article in Chinese). Chin J Neurol. 2010; 43(2): 146-153.

15. Liao HS, Zhu PL. Rehabilitation Assessment and Treatment of Stroke (published in Chinese). Beijing: Huaxia Publishing House; 1996: 149-150.

16. World Federation of Acupuncture-Moxibustion Societies (WFAS). Auricular Acupuncture Point (WFAS STANDARD-002: 2012). World Journal of Acupuncture-Moxibustion. 2013; 23(3): $12-21$. 
17. Treede RD, Rief W, Barke A, et al. Chronic pain as a symptom or a disease: The IASP Classification of Chronic Pain for the International Classification of Diseases (ICD-11). Pain. 2019; 160 (1): 19-27. doi: 10.1097/j.pain.0000000000001384

18. Gladstone DJ, Danells CJ, Black SE. The fugl-meyer assessment of motor recovery after stroke: a critical review of its measurement properties. Neurorehabil Neural Repair. 2002;16(3):232-240. doi:10.1177/154596802401105171

19. Mahoney FI, Barthel DW. Functional evaluation: the Barthel Index. Md State Med J. 1965; 14:61-5.

20. Shah S, Vanclay F, Cooper B. Improving the sensitivity of the Barthel Index for stroke rehabilitation. J Clin Epidemiol. 1989;42(8):703-709. doi:10.1016/0895-4356(89)90065-6

21. Ohura T, Hase K, Nakajima Y, Nakayama T. Validity and reliability of a performance evaluation tool based on the modified Barthel Index for stroke patients. BMC Med Res Methodol. 2017;17(1):131. Published 2017 Aug 25. doi:10.1186/s12874-017-0409-2

22. Zhao H, Nie WB, Sun YX, et al. Warm Needling Therapy and Acupuncture at Meridian-Sinew Sites Based on the Meridian-Sinew Theory: Hemiplegic Shoulder Pain. Evid Based Complement Alternat Med. 2015; 2015:694973. doi: 10.1155/2015/694973

23. Zheng J, Wu Q, Wang $\mathrm{L}$, et al. A clinical study on acupuncture in combination with routine rehabilitation therapy for early pain recovery of post-stroke shoulder-hand syndrome. Exp Ther Med. 2018; 15(2): 2049-2053. doi: 10.3892/etm.2017.5659

24. Ju ZY, Wang K, Cui HS, et al. Acupuncture for neuropathic pain in adults. Cochrane Database Syst Rev. 2017; 12(12):CD012057. doi: 10.1002/14651858.CD012057.pub2

25. Li RW, Guo J, Dou J, et al. Effect of the contralateral needling therapy on post-stroke shoulder-hand syndrome (article in Chinese). Acupuncture Research. 2020; 45(2):152-156.

26. Schulz KF, Altman DG, Moher D. CONSORT 2010 statement: updated guidelines for reporting parallel group randomized trials. BMC Med. 2010; 8(1): 18.doi: 10.1186/1741-7015-8-18

27. Lesaffre E. Superiority, equivalence, and non-inferiority trials. Bull NYU Hosp Jt Dis. 2008;66(2):150154.

28. Sheng YQ, Wu XL. Nearly Five Years of Chinese and Western Medicine Research Progress in the Treatment of Shoulder Hand Syndrome after Stroke (article in Chinese). Clinical journal of traditional Chinese medicine. 2016; 28(2):282-284.

29. Alimi D, Rubino C, Pichard-Léandri E, Fermand-Brulé S, Dubreuil-Lemaire ML, Hill C. Analgesic effect of auricular acupuncture for cancer pain: a randomized, blinded, controlled trial. J Clin Oncol. 2003;21(22):4120-4126. doi:10.1200/JC0.2003.09.011

30. Crew KD, Capodice JL, Greenlee H, et al. Randomized, blinded, sham-controlled trial of acupuncture for the management of aromatase inhibitor-associated joint symptoms in women with early-stage breast cancer. J Clin Oncol. 2010;28(7):1154-1160. doi:10.1200/JC0.2009.23.4708

\section{Figures}




\begin{tabular}{|c|c|c|c|c|c|c|}
\hline \multirow[b]{3}{*}{ TIMEPOINT } & \multicolumn{6}{|c|}{ STUDY PERIOD } \\
\hline & \multirow{2}{*}{$\begin{array}{c}\text { Enrollment } \\
-t_{1} \\
\end{array}$} & \multirow{2}{*}{$\begin{array}{c}\text { Allocation } \\
0 \\
\end{array}$} & \multirow{2}{*}{$\begin{array}{c}\text { Treatment } \\
t_{1} \\
\end{array}$} & \multicolumn{3}{|c|}{ Follow-ups } \\
\hline & & & & $t_{2}$ & $t_{3}$ & $\mathrm{t}_{4}$ \\
\hline \multirow{4}{*}{$\begin{array}{r}\text { ENROLLMENT } \\
\text { Eligibility checking } \\
\text { Sign of informed consent } \\
\text { Allocation }\end{array}$} & & & & & & \\
\hline & $x$ & & & & & \\
\hline & $x$ & & & & & \\
\hline & & $x$ & & & & \\
\hline \multicolumn{7}{|l|}{ INTERVENTIONS } \\
\hline Group A (BA) & & & $x$ & & & \\
\hline Group B (BA+ sham AA) & & & $x$ & & & \\
\hline Group $\mathrm{C}(\mathrm{BA}+\mathrm{AA})$ & & & $x$ & & & \\
\hline \multicolumn{7}{|l|}{ ASSESSMENTS } \\
\hline \multirow{3}{*}{$\begin{array}{r}\text { Demography } \\
\text { Cerebral CT or MRI } \\
\text { VAS }\end{array}$} & $x$ & & & & & \\
\hline & $x$ & & & & & \\
\hline & $x$ & & $x$ & $x$ & $x$ & $\times$ \\
\hline \multirow{3}{*}{$\begin{array}{r}\text { ULFMA } \\
\text { MBI } \\
\text { Adverse Events }\end{array}$} & $x$ & & $x$ & $x$ & $x$ & $\times$ \\
\hline & $x$ & & $x$ & $x$ & $x$ & $x$ \\
\hline & & & $x$ & $x$ & $x$ & $x$ \\
\hline
\end{tabular}

Figure 1

Trial Schedule 


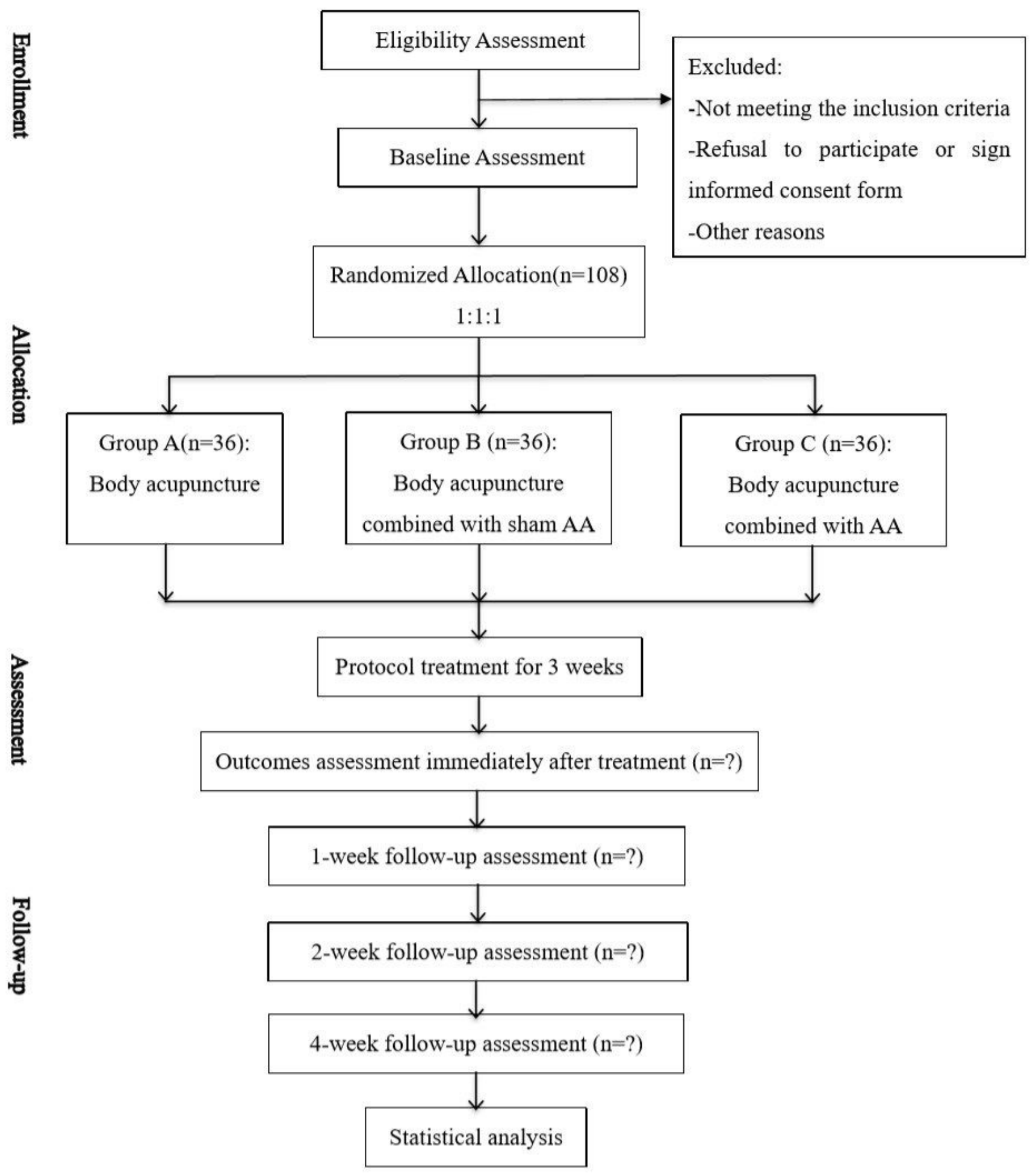

Figure 2

Trial flow chart 

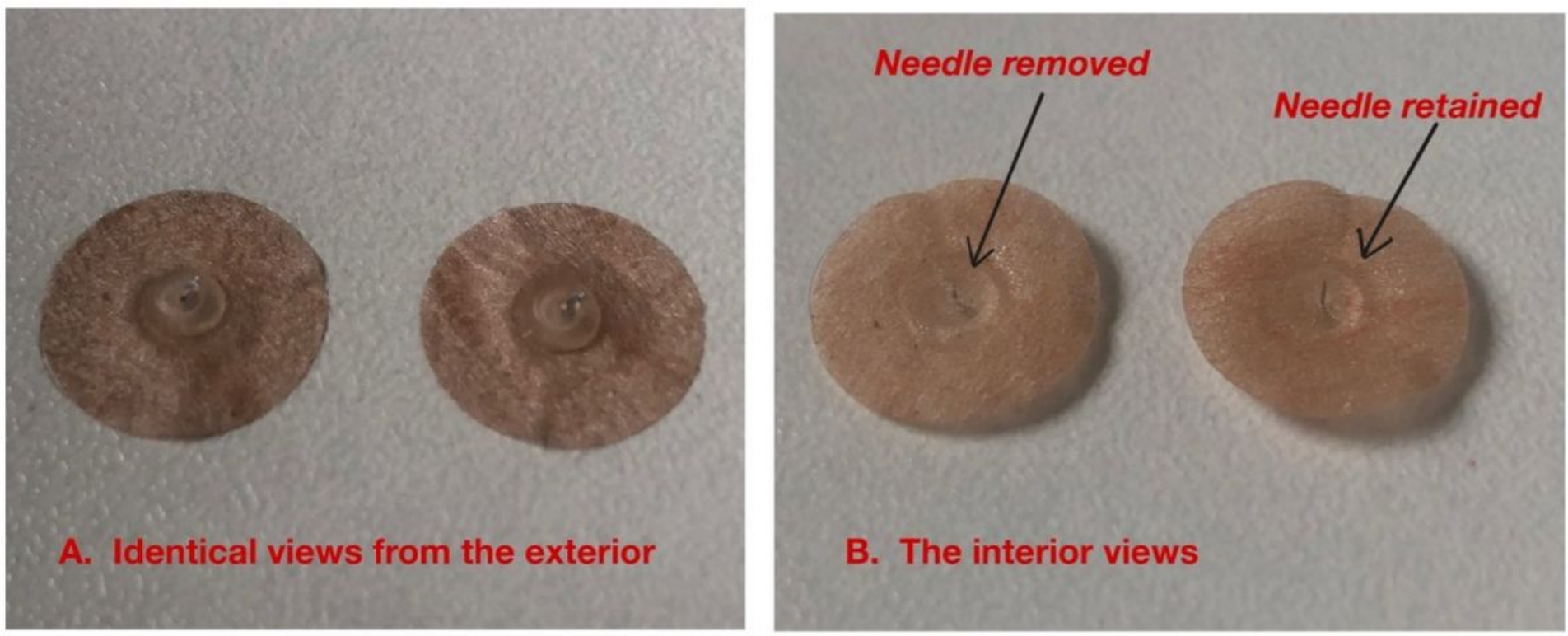

\section{Figure 3}

The exterior and the interior views of auricular needles

\section{Supplementary Files}

This is a list of supplementary files associated with this preprint. Click to download.

- SPIRITchecklist20200905.docx 